\title{
Comparison of characteristics during backward walking according to various stride frequencies in underwater and ground environments
}

\author{
Heejoong Kim ${ }^{a}$, Yijung Chung ${ }^{b}$ \\ ${ }^{a}$ Department of Physical Therapy, Bobath Memorial Hospital, Seongnam, Republic of Korea \\ ${ }^{b}$ Department of Physical Therapy, College of Health Science and Social Welfare, Sahmyook University, Seoul, Republic of Korea
}

Objective: The purpose of this study was to investigate the relationship between heart rate (HR), self-awareness of exercise intensity (rating of perceived exertion, RPE), and 5-meter walk test (5MWT) of persons affected by stroke during backward walking according to the preferred stride frequency (PSF), PSF +3 and PSF +6 conditions.

Design: Cross-sectional study.

Methods: A total of 11 persons with stroke ( 9 males, 2 females) participated voluntarily. All patients underwent backward walking under the PSF, PSF +3 , and PSF +6 conditions in underwater and ground environments, and each condition was performed for 5 minutes. The HR, RPE, and walking speeds were measured during walking, and the measured values from underwater and ground environments were compared.

Results: The HR and RPE in the ground environment were significantly increased $(p<0.05)$, and although the 5MWT showed an increase in speed, it was not significant. The HR and RPE in the underwater environment were also significantly increased $(p<0.05)$, however, although the 5MWT results was increased, it was not significant. The HR and RPE were significantly increased in the PSF +6 condition $(p<0.05)$.

Conclusions: The results of this study showed that backward gait training underwater can provide an appropriate exercise intensity for stroke survivors and suggests that exercises performed in an underwater environment is more effective compared to the ground environment.

Key Words: Backward locomotion, Gait, Immersion, Stroke

\section{Introduction}

Restoration of walking ability is an important factor in quality of life and functional independence of daily living in persons with stroke, and it is the most important goal in post-stroke rehabilitation [1]. Various treatment methods have been suggested in order to improve gait, and backward walking has been recommended for persons with hemiplegia for appropriate adjustment of exercise and enhancement of walking activities [2].
Other studies have demonstrated that asymmetrical gait patterns in stroke survivors can be improved [3], leading to increased walking speed [4]. Recently, it has been found that muscular activity and movement mechanisms are mediated by backward gait in healthy adults $[5,6]$. Another method of exercise for stroke survivors is water exercise, which is less burdensome on the lower extremities. It uses water buoyancy and resistance to improve muscle strength, muscle endurance, balance ability, cardiopulmonary endurance, etc [7]. Exercising underwater provides physical and psycho-

Received: 24 May, 2018 Revised: 10 June, 2018 Accepted: 15 June, 2018

Corresponding author: Yijung Chung (ORCID http://orcid.org/0000-0002-2431-8895)

Department of Physical Therapy, College of Health Science and Social Welfare, Sahmyook University, 815 Hwarang-ro, Nowon-gu, Seoul 01795, Republic of Korea

Tel: 82-2-3399-1637 Fax: 82-2-3399-1639 E-mail: yijung36@syu.ac.kr

(c) This is an Open-Access article distributed under the terms of the Creative Commons Attribution Non-Commercial License (http://creativecommons.org/licenses/ by-nc/4.0) which permits unrestricted non-commercial use, distribution, and reproduction in any medium, provided the original work is properly cited.

Copyright $@ 2018$ Korean Academy of Physical Therapy Rehabilitation Science 
logical stability, which may further allow stroke survivors to perform exercises with increased confidence and observe a greater exercise effect. Studies on backward walking in an underwater environment are increasing and have shown that backward walking in water may lead to greater muscle activity in the paraspinal muscles [8], compared to the ground environment [9]. In addition, compared to the forward, the backward gait showed a greater metabolic rate and the kinesthetic angle was also higher [10]. Recently, we measured the metabolic expenditure and the exercise angle by comparing backward gait in the underwater and ground environment. Unlike forward gait, which is set at half of the ground, half-speed adjustment in the backward gait was considered inappropriate to induce a similar metabolic depletion and rating of perceived exertion (RPE) [11]. Other studies have also reported differences in spatio-temporal characteristics and joint angles during forward and backward gait [12]. However, previous studies have shown that most of the studies on underwater walking training are mainly for healthy adults, and studies on underwater walking training for stroke survivors are insufficient. In addition, studies focused on backward walking training in underwater environments for stroke survivors are also lacking. Therefore, the purpose of this study was to investigate the physiological and selfawareness changes according to the stride frequency of persons with stroke during backward gait in underwater and ground environments.

\section{Methods}

\section{Subjects}

Patients who were admitted to Bobath Memorial Hospital in Seongnam city, Gyeonggi-do for stroke who fulfilled the following conditions were included in the study: those who had been diagnosed with stroke 6 months ago or more, a Mini-Mental State Examination score of 24 or more, no serious cognitive impairment, those who can perform the evaluation according to the direction of the researcher, those who can walk more than 10 meters independently, those who do not have cardiopulmonary or orthopedic diseases, and those without hearing impairments. All subjects signed a consent form, and all procedures in this study were approved by the Sahmyook University Institutional Review Board (IRB No. 2-7001793-AB-N-012018067HR).

\section{Methods}

Prior to the start of the experiment, all of the subjects had
Table 1. General characteristics of the subjects $(\mathrm{N}=11)$

\begin{tabular}{lc}
\hline Characteristic & Value \\
\hline Sex (male/female) & $9 / 2$ \\
Age $(\mathrm{y})$ & $45.55(17.33)$ \\
Height $(\mathrm{cm})$ & $169.27(9.05)$ \\
Weight $(\mathrm{kg})$ & $70.38(14.38)$ \\
MMSE-K & $26.91(1.92)$ \\
\hline
\end{tabular}

Values are presented as number only or mean (SD). MMSE-K: Korean version of Mini-Mental State Examination.

adequate amount of practice in order to be able to proceed with the actual measurements. In addition, all subjects fully understood about having self-awareness of the exercise intensity prior to the assessment, and the physiological data was collected before each experiment (Table 1).

During the experiment, a therapist accompanied the patient to prevent falls. At the start of the experiment, subjects began walking at their most comfortable speed, and increases in speed was based on Masumoto's method [13]. However, PSF of +3 and +6 , respectively. If it is difficult to increase the speed, it is set to be lower. The experiment was carried out for 5 minutes per stride frequency. Heart rate (HR), exercise intensity (RPE), and 5-meter walk test (MWT) were measured during the last remaining 5 minutes. The HR was measured using a handheld device (POLAR RS400sd; Polar Electro, Kempele, Finland) and the trainer angle was Borg's RPE 6 to 20 scale [14]. Subjects were provided with a 1-minute rest period between the three different speeds. Subjects walked on ground or underwater randomly and were provided with as 30-minute rest period between each environment [11]. The training in the underwater environment was carried out in a pool with the dimenstions of 6 meters in width, 4 meters in length, 1.2 meters in depth, and the water temperature was $32^{\circ} \mathrm{C} \pm 1^{\circ} \mathrm{C}$ (Figure 1).

All data was analyzed using the statistical program IBM SPSS Statistics ver. 20.0 (IBM Co., Armonk, NY, USA), and repeated measures analysis of variance was used to investigate the difference of variables according to the different periods. In addition, an independent sample t-test was conducted to compare the differences between the underwater and the ground environment. All statistical significance levels were set at $p<0.05$.

\section{Results}

The ground environment HR was $74.36 \pm 13.02$ in the PSF, $76.36 \pm 13.87$ in the PSF +3 , and $80.36 \pm 13.22$ in the PSF + 
6 , and showed a statistically significant difference. The ground environment RPE was $10.55 \pm 1.81$ in the PSF, $12.64 \pm 1.96$ in the PSF +3 , and $13.91 \pm 1.87$ in the PSF +6 , and showed a statistically significant difference. The ground environment 5MWT showed $41.01 \pm 30.11$ in the PSF, $37.69 \pm 27.16$ in the PSF +3 , and $31.96 \pm 18.29$ in the PSF +

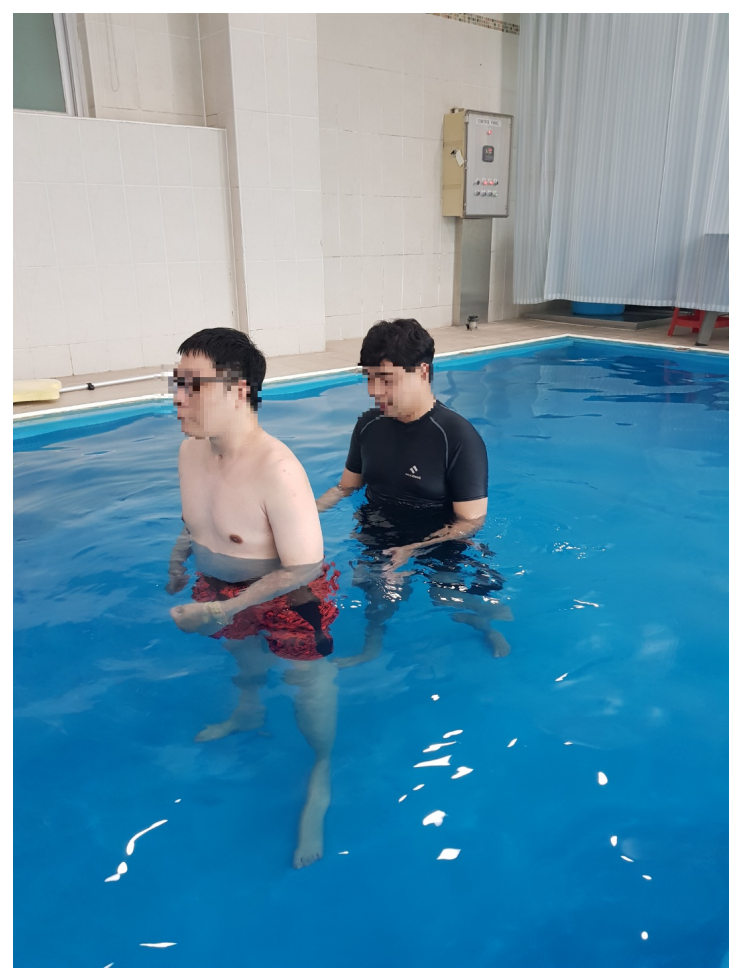

Figure 1. Backward walking in water.
6 , but the difference was not statistically significant. The underwater environment HR showed $80.09 \pm 12.90$ in PSF, 85.91 \pm 12.39 in PSF +3 , and $92.00 \pm 12.33$ in $\mathrm{PSF}+6$, and showed a statistically significant difference. The underwater environment RPE was $10.45 \pm 1.70$ in PSF, $13.00 \pm 1.55$ in $\mathrm{PSF}+3$, and $16.09 \pm 0.94$ in PSF +6 , and showed a statistically significant difference. The underwater $5 \mathrm{MWT}$ was $27.24 \pm 12.75$ in the PSF, $24.24 \pm 12.23$ in the PSF +3 , and $22.19 \pm 12.43$ in the PSF +6 , but the difference was not statistically significant. When comparing the characteristics between the two environments, HR in underwater walking was significantly faster than that in PSF +6 , and RPE in underwater walking was also significantly higher in $\mathrm{PSF}+6$. The 5MWT showed no significant difference between the two groups (Table 2).

\section{Disscussion}

Human walking is a fundamental component functional independence that is generally affected by disease or injury, and is therefore considered to be an ultimate goal in most rehabilitation processes [15]. In this study, we investigated changes in HR, RPE, and walking speed with backward gait according to changes in stride frequency in underwater and ground environment. In the aquatic environment, the HR and RPE were higher compared to the ground environment, which supports the results from previous studies [16-18].

It was also consistent with previous assertions in which higher metabolic expenditure and higher exercise angles

Table 2. HR, RPE, walking speeds according to the various environments and stride frequencies

$(\mathrm{N}=11)$

\begin{tabular}{|c|c|c|c|c|c|}
\hline \multirow{2}{*}{ Variable } & \multicolumn{3}{|c|}{ Stride frequency } & \multirow{2}{*}{$\mathrm{F}$} & \multirow{2}{*}{$p$-value } \\
\hline & PSF & $\mathrm{PSF}+3$ & $\mathrm{PSF}+6$ & & \\
\hline \multicolumn{6}{|l|}{ HR } \\
\hline Land & $74.36(13.02)$ & $76.36(13.87)$ & $80.36(13.22)$ & 39.487 & $<0.001$ \\
\hline Water & 80.09 (12.90) & 85.91 (12.39) & $92.00(12.33)$ & 30.982 & $<0.001$ \\
\hline$p$-value & 0.312 & 0.104 & $0.045^{*}$ & & \\
\hline \multicolumn{6}{|l|}{ RPE } \\
\hline Land & $10.55(1.81)$ & $12.64(1.96)$ & $13.91(1.87)$ & 29.00 & $<0.001$ \\
\hline Water & $10.45(1.70)$ & $13.00(1.55)$ & $16.09(0.94)$ & 124.655 & $<0.001$ \\
\hline$p$-value & 0.094 & 0.635 & 0.002 & & \\
\hline \multicolumn{6}{|l|}{$5 \mathrm{MWT}$} \\
\hline Land & $41.01(30.11)$ & $37.69(27.16)$ & $31.96(18.29)$ & 4.325 & 0.064 \\
\hline Water & $27.24(12.75)$ & $24.24(12.23)$ & $22.19(12.43)$ & 2.551 & 0.103 \\
\hline$p$-value & 0.178 & 0.150 & 0.158 & & \\
\hline
\end{tabular}

Values are presented as mean (SD).

HR: heart rate, RPE: rating of perceived exertion, PSF: perferred stride frequency, 5 MWT: 5 meter walk test. 
were associated with greater stride frequency (SF) [13]. In addition, we could observe that, although it was not statistically significant, the PSF was slower in the underwater compared to the ground environment, and the HR and RPE were increased with increasing SF. Particularly, it should be noted that HR and RPE were significantly increased in the underwater environment compared to the ground environment at the PSF +6 point. These symptoms were reported to be due to higher load on the joints due to increased underwater viscous resistance when performing fast underwater walking in the past [19].

However, recent research by Masumoto et al. [11] showed that at higher speeds, the backward walking on the ground environment produced higher metabolic depletion and RPE comapred to the aquatic environment. We conclude that our results were due to the fact that this study involved walking on the ground rather than the treadmill, unlike the study of Masumoto et al. [11]. Therefore, further studies examining the characteristics and differences in underwater treadmill walking and underwater walking are necessary. The reason why the increase in walking speed did not show a significant difference is considered to be due to the fact that persons with stroke have impaired visual acuity and therefore have to rely solely on their senses, and when their senses are also impaired, a certain activity can be unfamiliar and is difficult to perform. In addition, since the measurements were made during the last minute of the 5 minutes of training, it is considered that the physical strength and speed may have been affected by the constant underwater resistance. In this study, we investigated the effects of performing backward gait in an underwater environment on stroke survivors, but the information is not sufficient compared to that on forward gait and walking on a ground environment for persons with stroke. In future studies, it is necessary to find a more effective gait training method for those affected by stroke through biomechanical analysis, such as the study of muscle activity during backward walking in an underwater environment.

The results of this study show that the exercise period can be used to adjust the exercise intensity appropriate for persons with stroke during backward gait training in the water, and suggest that the underwater environment is more effective than the ground environment.

\section{Conflict of Interest}

The authors declared no potential conflicts of interest with respect to the authorship and/or publication of this article.

\section{References}

1. Kelly-Hayes M, Beiser A, Kase CS, Scaramucci A, D'Agostino $\mathrm{RB}$, Wolf PA. The influence of gender and age on disability following ischemic stroke: the Framingham study. J Stroke Cerebrovasc Dis 2003;12:119-26.

2. Bobath B. Adult hemiplegia: evaluation and treatment. Oxford: Butterworth/Heinemann; 1990.

3. Yang YR, Yen JG, Wang RY, Yen LL, Lieu FK. Gait outcomes after additional backward walking training in patients with stroke: a randomized controlled trial. Clin Rehabil 2005;19:264-73.

4. Takami A, Wakayama S. Effects of partial body weight support while training acute stroke patients to walk backwards on a treadmill-a controlled clinical trial using randomized allocation. J Phys Ther Sci 2010;22:177-87.

5. Lee M, Kim J, Son J, Kim Y. Kinematic and kinetic analysis during forward and backward walking. Gait Posture 2013;38:674-8.

6. Mahaki M, De Sá E Souza GS, Mimar R, Vieira MF. The comparison of ground reaction forces and lower limb muscles correlation and activation time delay between forward and backward walking. Gait Posture 2017;58:380-5.

7. Bates A, Hanson N. The principles and properties of water. Aquatic exercise therapy Philadelphia: WB Saunders. 1996: $1-320$.

8. Masumoto K, Takasugi S, Hotta N, Fujishima K, Iwamoto Y. Muscle activity and heart rate response during backward walking in water and on dry land. Eur J Appl Physiol 2005;94:54-61.

9. Masumoto K, Takasugi S, Hotta N, Fujishima K, Iwamoto Y. A comparison of muscle activity and heart rate response during backward and forward walking on an underwater treadmill. Gait Posture 2007;25:222-8.

10. Masumoto K, Hamada A, Tomonaga HO, Kodama K, Amamoto Y, Nishizaki Y, et al. Physiological and perceptual responses to backward and forward treadmill walking in water. Gait Posture 2009;29:199-203.

11. Masumoto K, Hamada A, Tomonaga HO, Kodama K, Amamoto Y, Nishizaki Y, et al. Metabolic costs and rating of perceived exertion during backward walking in water and on dry land. Res Sports Med 2015;23:27-36.

12. Cadenas-Sánchez C, Arellano R, Taladriz S, López-Contreras G. Biomechanical characteristics of adults walking forward and backward in water at different stride frequencies. J Sports Sci 2016;34:224-31.

13. Masumoto K, Nishizaki Y, Hamada A. Effect of stride frequency on metabolic costs and rating of perceived exertion during walking in water. Gait Posture 2013;38:335-9.

14. Borg GA. Psychophysical bases of perceived exertion. Med Sci Sports Exerc 1982;14:377-81.

15. Turnbull GI, Charteris J, Wall JC. A comparison of the range of walking speeds between normal and hemiplegic subjects. Scand J Rehabil Med 1995;27:175-82.

16. Hall J, Macdonald IA, Maddison PJ, O’Hare JP. Cardiorespiratory responses to underwater treadmill walking in healthy females. Eur J Appl Physiol Occup Physiol 1998;77:278-84. 
17. Masumoto K, Shono T, Hotta N, Fujishima K. Muscle activation, cardiorespiratory response, and rating of perceived exertion in older subjects while walking in water and on dry land. J Electromyogr Kinesiol 2008;18:581-90.

18. Chu KS, Eng JJ, Dawson AS, Harris JE, Ozkaplan A, Gylfadóttir S. Water-based exercise for cardiovascular fitness in people with chronic stroke: a randomized controlled trial. Arch Phys Med Rehabil 2004;85:870-4.

19. Miyoshi T, Shirota T, Yamamoto S, Nakazawa K, Akai M. Functional roles of lower-limb joint moments while walking in water. Clin Biomech (Bristol, Avon) 2005;20:194-201. 